\title{
Mechanical Problem Identified
}

National Cancer Institute

\section{Source}

National Cancer Institute. Mechanical Problem Identified. NCI Thesaurus. Code C92079.

Problems that result from internal or external forces including fluids, other objects, or environmental or physiologic influences. 